\title{
Respiratory Syncytial Virus Infection in Homeless Populations, Washington, USA
}

\section{Jim Boonyaratanakornkit, ${ }^{1}$ Seda Ekici, ${ }^{1}$ Amalia Magaret, Kathryn Gustafson, Emily Scott, Micaela Haglund, Jane Kuypers, Ronald Pergamit, John Lynch, Helen Y. Chu}

Homelessness has not previously been identified as a risk factor for respiratory syncytial virus (RSV) infection. We conducted an observational study at an urban safety-net hospital in Washington, USA, during 2012-2017. Hospitalized adults with RSV were more likely to be homeless, and several clinical outcome measures were worse with RSV than with influenza.

$\mathrm{R}$ espiratory syncytial virus (RSV) is increasingly recognized as a major pathogen in adults and shows a disease burden comparable to that for influenza (1). No vaccine is currently available. However, several vaccine candidates against RSV are in clinical trials, and the elderly, those with chronic lung disease, infants, and immunocompromised persons remain priority target populations for prevention efforts $(2,3)$.

Rates of homelessness are increasing in major urban centers because of lack of affordable housing and slower wage growth (4). Homeless persons experience higher rates of illness and death compared with the general population, partly because of infectious diseases from lack of access to sanitation, crowding in shelters, untreated chronic medical conditions, and higher rates of mental health issues and substance use (5). Studies have described local outbreaks of rhinovirus and influenza in homeless shelters (6). Identification of homeless persons as an at-risk population for severe RSV disease might guide prioritization strategies for RSV vaccines and therapeutics as they become available. We aimed to evaluate risk factors and clinical outcomes of adults hospitalized with RSV infections versus those with influenza in an urban medical center serving a region that had high rates of homelessness.

Author affiliations: University of Washington, Seattle, Washington, USA (J. Boonyaratanakornkit, S. Ekici, A. Magaret, K. Gustafson,

E. Scott, M. Haglund, J. Kuypers, J. Lynch, H.Y. Chu); Harborview Medical Center, Seattle (J. Boonyaratanakornkit, R. Pergamit)

\section{The Study}

We conducted a retrospective case-control study of adults hospitalized with RSV and influenza at Harborview Medical Center (Seattle, WA, USA) during July 2012-June 2017. This center is an academic tertiary medical center that functions as the safety-net hospital for the Seattle metropolitan area. We identified patients on the basis of laboratory records of specimens containing influenza A/B virus and RSV by using a rapid PCR assay (Focus Diagnostics, https://www. focusdx.com) or the Xpert Xpress Flu/RSV test (Cepheid, http://www.cepheid.com) (Appendix, https://wwwnc.cdc. gov/EID/article/25/7/18-1261-App1.pdf).

A total of 865 patients were hospitalized with RSV infection $(\mathrm{n}=157)$ or influenza A/B $(\mathrm{n}=708)$ during July 2012-June 2017 (Table 1; Figure 1). We showed by multivariable analysis of risk factors for hospitalization with RSV infection versus influenza that older age, homelessness, having chronic obstructive pulmonary disease (COPD) or asthma, and drug use were associated with an increased odds ratio (OR) for RSV hospitalization compared with influenza (Table 1; Figure 2). Drug use showed a correlation with homelessness (OR 5.18, 95\% CI 3.17-8.46).

Overall, a higher proportion of adults hospitalized with RSV infection were admitted to the intensive care unit (ICU), readmitted within 30 days, and received any antimicrobial drug compared with patients hospitalized with influenza (Table 2). A total of $4 \%(7 / 158)$ of adults given a diagnosis of RSV infection died during hospitalization, compared with $3 \%(21 / 712)$ of those with influenza. Having COPD/asthma was not correlated with antimicrobial drug use (OR 1.07, 95\% CI 0.71-1.60). Only 10\% (4/40) of patients with RSV infection who were readmitted within 30 days had a positive swab specimen for the same virus at the second admission.

We sought to determine whether increased hospital readmission after hospitalization for RSV infection had other potential explanatory factors. We found by multivariable analysis that having RSV infection (OR 2.40, 95\% CI 1.54-3.76) and homelessness (OR 2.06, 95\% CI 1.31-3.24) remained associated with an increased odds of hospital readmission. Because homelessness and RSV infection increased the odds of readmission, persons at highest risk were homeless persons with RSV infection (OR 2.4

${ }^{1}$ These authors contributed equally to this article. 
Table 1. Analysis of sociodemographic characteristics of patients admitted with RSV infection or influenza, Washington, USA, 2012-2017*

\begin{tabular}{|c|c|c|c|c|c|}
\hline Characteristic & $\mathrm{RSV}, \mathrm{n}=157$ & Influenza, $\mathrm{n}=708$ & $\begin{array}{c}\text { Univariable } \\
\text { p value }\end{array}$ & $\begin{array}{c}\text { RSV vs. influenza, OR } \\
(95 \% \mathrm{Cl})\end{array}$ & $\begin{array}{c}\text { Multivariable } \\
\text { p value }\end{array}$ \\
\hline Male sex & $100(64)$ & $433(61)$ & 0.590 & - & - \\
\hline Black or African American & $26(17)$ & $201(28)$ & 0.002 & - & - \\
\hline Homeless & $50(32)$ & 137 (19) & $<0.001$ & $2.00(1.33-3.03)$ & 0.001 \\
\hline Drug use & $26(17)$ & $60(8)$ & 0.005 & $1.79(1.06-3.03)$ & 0.028 \\
\hline Asthma/COPD & $32(20)$ & $95(13)$ & 0.034 & $1.67(1.06-2.63)$ & 0.027 \\
\hline
\end{tabular}

$\times 2.06=4.95$ relative to housed persons with influenza). Age (OR 1.00, 95\% CI 0.99-1.02), having COPD/asthma (OR 0.88, 95\% CI 0.47-1.58), and drug use (OR 1.15, 95\% CI 0.62-2.13) were not correlated with readmission.

We found that $6.5 \%(24,452 / 374,672)$ of all patients discharged from Harborview Medical Center during 2012 2017 were homeless. In that same period, 32\% (50/157) of those with RSV infection were homeless, compared with $19 \%(147 / 708)$ of those with influenza $(p=0.003)$, $3.4 \%(286 / 8,488)$ of patients with a urinary tract infection $(\mathrm{p}<0.001)$, and $2.0 \%(25 / 1,278)$ of patients with an ischemic stroke $(\mathrm{p}<0.001)$.

\section{Conclusions}

In this study of adults hospitalized during 5 years in an urban hospital, $32 \%$ of patients given a diagnosis of RSV infection were homeless, compared with $6.5 \%$ of all patients hospitalized. Patients hospitalized with RSV infection were more likely to be older, homeless, drug users, or have COPD/asthma compared with persons with influenza. Homelessness has reached a national public health crisis, and many homeless persons seek acute care in emergency departments (9). The city of Seattle has the largest concentration of homelessness per capita in the country (10). Outbreaks of infections with respiratory viruses have been described in homeless shelters, in which transmission might be facilitated by crowding, poor sanitation, and the ability of RSV to spread through fomites $(6,11)$. Our findings for $\mathrm{RSV}$ in this homeless population might be generalizable to other urban public hospitals.

Several outcome measures were worse in patients hospitalized for RSV infection than for influenza, including 30-day readmission, admission to the ICU, and receipt of antimicrobial drugs. A previous study similarly reported higher rates of ICU admission among patients with RSV infection than for those with influenza (12). More severe disease might have led clinicians to preferentially use antimicrobial drugs for patients hospitalized with RSV infection compared with influenza. The higher number of patients with RSV infection than influenza admitted to the ICU supports this interpretation. These results suggest that patients hospitalized with RSV infection might benefit from closer monitoring, follow-up, and antimicrobial drug stewardship to prevent readmission and overuse of antimicrobial drugs.

Homelessness and having RSV infection were independent risk factors for hospital readmission, demonstrating that the higher risk for poor outcomes in homeless persons was not simply explained by the disproportionately higher number of diagnosis of RSV infection in this group. All-cause readmission within 30 days is a major quality metric used by the Centers for Medicare and Medicaid

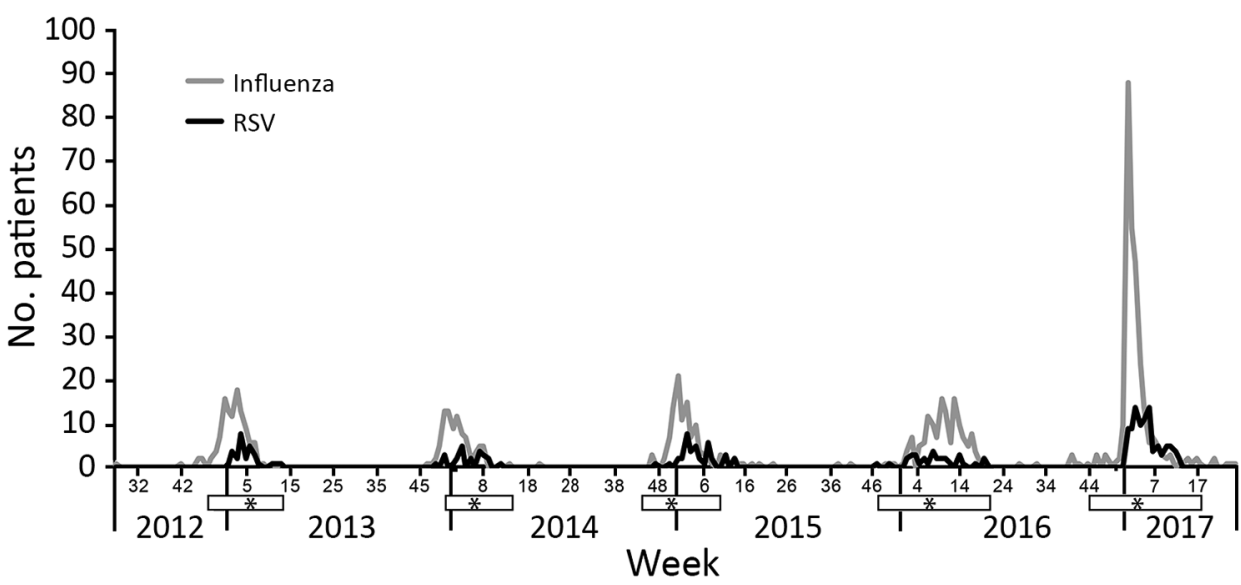

Figure 1. Detection of influenza and RSV in adults hospitalized at Harborview Medical Center, Seattle, WA, USA, July 2012June 2017. White bars below the $\mathrm{x}$-axis indicate RSV seasons; asterisks indicate weeks when cases of RSV infection peaked, on the basis of Centers for Disease Control and Prevention surveillance data in region 10 (Alaska, Idaho, Washington, and Oregon) $(7,8)$ during 2012-2017. RSV, respiratory syncytial virus. 

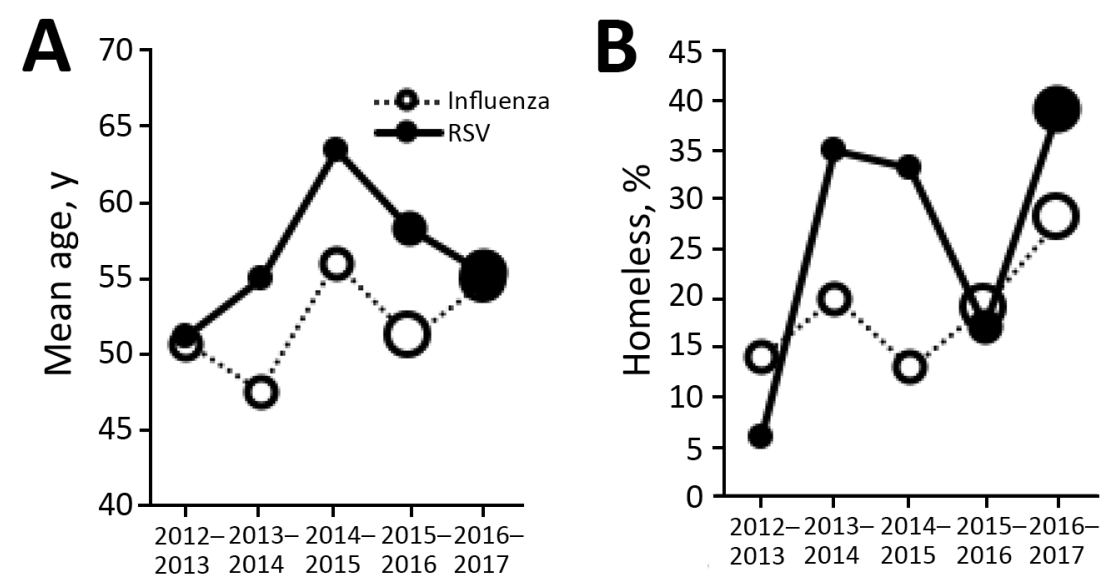

Figure 2. Sociodemographic characteristics of patients hospitalized with RSV infection or influenza across 5 seasons, 2012-2017, Washington, USA. A) Age; B) homelessness; C) drug use; and D) COPD or asthma. Size of each circle indicates number of patients for that data point: small circles indicate $<50$ patients, medium circles indicate 50-150 patients, and large circles indicate $>150$ patients. COPD, chronic obstructive pulmonary disease; RSV, respiratory syncytial virus.
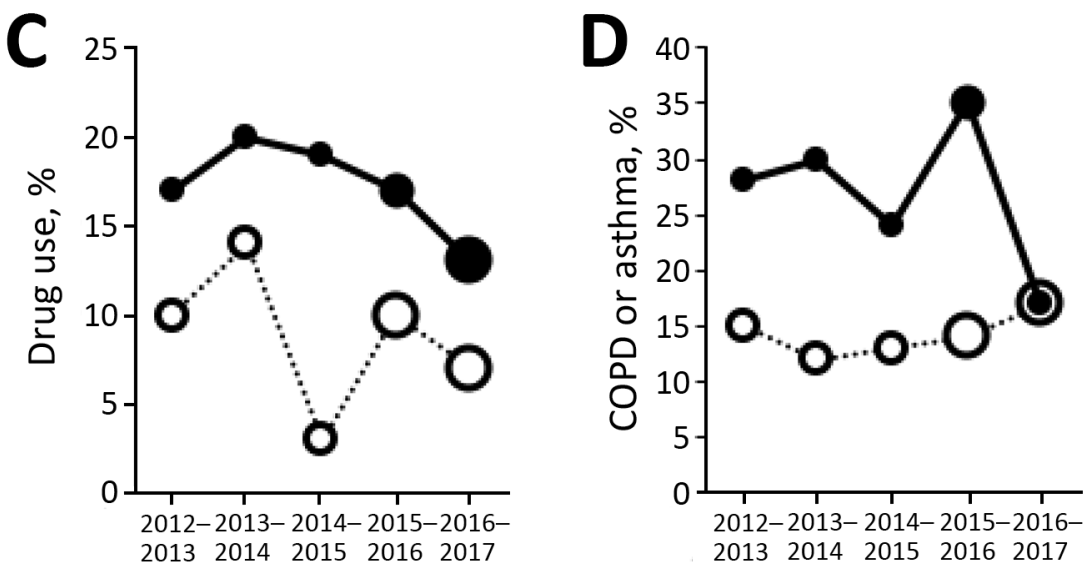

Services and Hospital Quality Alliance (http://www.allhealthpolicy.org/glossary/hospital-quality-alliance). Other studies have found an association between lower education and unemployment with rehospitalization and that most rehospitalizations were related to concurrent conditions (13).

Limitations of this study include the retrospective study design, limiting analysis to only hospitalized patients with RSV infection or influenza. The overall number of hospitalizations annually for RSV infection and influenza increased during 2012-2017 and was likely caused by transition to on-site rapid testing, which might increase provider uptake (14). In addition, without hospitalized and community controls who do not have influenza or RSV infection and who are not homeless, we cannot definitively conclude that homelessness is associated with a greater risk for hospitalization for RSV infection compared with influenza. The association might have 3 possible interpretations: risk factors are associated with more severe disease caused by RSV than influenza; risk factors are associated with a higher risk for infection with RSV compared with influenza; or risk factors are associated with greater susceptibility to RSV infection and disease compared with influenza. In addition, this study was limited to a single site, although it is representative of public, safety-net hospitals. Additional limitations include clinician-initiated testing triggered by influenza-like symptoms rather than for detection of RSV

\begin{tabular}{|c|c|c|c|}
\hline Characteristic & RSV, $n=158$ & Influenza, $\mathrm{n}=712$ & Univariable $p$ value \\
\hline Mean length of hospital stay, $d$ & 5.5 & 4.6 & 0.67 \\
\hline ICU admission & $39(25)$ & $123(17)$ & 0.041 \\
\hline Mean length of ICU stay, $d$ & 3.5 & 3.6 & 0.86 \\
\hline Readmission within $30 \mathrm{~d}$ & $40(25)$ & $79(11)$ & $<0.001$ \\
\hline Patients fitting SIRS criteria at admission & $78(49)$ & $309(43)$ & 0.18 \\
\hline Antimicrobial drugs used $\dagger$ & $84(53)$ & $224(31)$ & $<0.001$ \\
\hline Steroids used & $22(14)$ & $61(9)$ & 0.05 \\
\hline Deaths & $7(4)$ & $21(3)$ & 0.61 \\
\hline
\end{tabular}

*Values are no. (\%) unless otherwise noted. Four patients were admitted twice with influenza, and 1 patient was admitted twice with RSV infection. ICU, intensive care unit; RSV, respiratory syncytial virus; SIRS, systemic inflammatory response syndrome.

†Included vancomycin, ceftriaxone, meropenem, azithromycin, levofloxacin, ciprofloxacin, amoxicillin, piperacillin/tazobactam, or ampicillin. 
infection, which is less likely to manifest with fever (15). Therefore, the true burden of RSV infection is likely higher than identified in this study.

In conclusion, homeless persons might represent a previously unrecognized population at increased risk for poor outcomes caused by infection with RSV. An effective vaccine or therapeutic in adults could benefit this medically underserved population. Further data on the impact of homelessness on respiratory virus infection severity and outcomes are needed to guide public health strategies and implementation.

\section{Acknowledgments}

We thank Ferric Fang and Tom Smith for providing assistance with obtaining residual nasal swabs specimens, and Larissa Lewis for reporting respiratory virus infections.

This study was supported by National Institutes of Health grants T32-AI007044 (to J.B.), T32-AI118690 (to J.B.), and K23-AI103105 (to H.Y.C.), and the University of Washington Chair of Medicine Scholars Award (to H.Y.C.).

H.Y.C. has received research support from GlaxoSmithKline, Novavax, Pfizer, and Sanofi.

\section{About the Author}

Dr. Boonyaratanakornkit is a senior fellow at the University of Washington and the Fred Hutchinson Cancer Research Center, Seattle, WA. His primary research interest is the epidemiology and immunology of respiratory viruses.

\section{References}

1. Falsey AR, Hennessey PA, Formica MA, Cox C, Walsh EE. Respiratory syncytial virus infection in elderly and high-risk adults. N Engl J Med. 2005;352:1749-59. http://dx.doi.org/10.1056/ NEJMoa043951

2. Falloon J, Yu J, Esser MT, Villafana T, Yu L, Dubovsky F, et al. An adjuvanted, postfusion F protein-based vaccine did not prevent respiratory syncytial virus illness in older adults. J Infect Dis. 2017;216:1362-70. http://dx.doi.org/10.1093/infdis/jix503

3. Higgins D, Trujillo C, Keech C. Advances in RSV vaccine research and development: a global agenda. Vaccine. 2016;34:2870-5. http://dx.doi.org/10.1016/j.vaccine.2016.03.109
4. Coleman V. Annual homeless count reveals more people sleeping outside than ever before. The Seattle Times. May 31, 2018 [cited 2018 May 31]. https://www.seattletimes.com/seattle-news/ homeless/new-homeless-count-in-king-county-shows-spike-innumber-of-people-sleeping-outside

5. Raoult D, Foucault C, Brouqui P. Infections in the homeless. Lancet Infect Dis. 2001;1:77-84. http://dx.doi.org/10.1016/ S1473-3099(01)00062-7

6. Hadler SC, Castro KG, Dowdle W, Hicks L, Noble G, Ridzon R. Epidemic intelligence service investigations of respiratory illness, 1946-2005. Am J Epidemiol. 2011;174(Suppl):S36-46. http://dx.doi.org/10.1093/aje/kwr309

7. Rose EB, Wheatley A, Langley G, Gerber S, Haynes A. Respiratory syncytial virus seasonality-United States, 2014-2017. MMWR Morb Mortal Wkly Rep. 2018;67:71-6. http://dx.doi.org/10.15585/ mmwr.mm6702a4

8. Haynes AK, Prill MM, Iwane MK, Gerber SI; Centers for Disease Control and Prevention. Respiratory syncytial virus - United States, July 2012-June 2014. MMWR Morb Mortal Wkly Rep. 2014;63:1133-6.

9. Donovan S, Shinseki EK. Homelessness is a public health issue. Am J Public Health. 2013;103(Suppl 2):S180.

10. Greenstone S. Seattle's homeless crisis the worst in the country? The Seattle Times. January 16, 2018 [cited 2019 Apr 3]. https://www.seattletimes.com/seattle-news/homeless/is-seattleshomeless-crisis-the-worst-in-the-country

11. Hall CB. The nosocomial spread of respiratory syncytial viral infections. Annu Rev Med. 1983;34:311-9. http://dx.doi.org/ 10.1146/annurev.me.34.020183.001523

12. Jasti H, Mortensen EM, Obrosky DS, Kapoor WN, Fine MJ. Causes and risk factors for rehospitalization of patients hospitalized with community-acquired pneumonia. Clin Infect Dis. 2008; 46:550-6. http://dx.doi.org/10.1086/526526

13. Ackerson B, Tseng HF, Sy LS, Solano Z, Slezak J, Luo Y, et al. Severe morbidity and mortality associated with respiratory syncytial virus versus influenza infection in hospitalized older adults. Clin Infect Dis. 2018; [Epub ahead of print]. http://dx.doi.org/10.1093/cid/ciy991

14. Chu HY, Englund JA, Huang D, Scott E, Chan JD, Jain R, et al. Impact of rapid influenza PCR testing on hospitalization and antiviral use: a retrospective cohort study. J Med Virol. 2015;87:2021-6. http://dx.doi.org/10.1002/jmv.24279

15. Colosia AD, Yang J, Hillson E, Mauskopf J, Copley-Merriman C, Shinde V, et al. The epidemiology of medically attended respiratory syncytial virus in older adults in the United States: A systematic review. PLoS One. 2017;12:e0182321. http://dx.doi.org/10.1371/ journal.pone. 0182321

Address for correspondence: Jim Boonyaratanakornkit, Harborview Medical Center, MS 359779, Seattle, WA 98104, USA; email: jboonyar@fredhutch.org 